\title{
Concretos com agregados reciclados de resíduos de construção e demolição e adições minerais: uma análise bibliográfica
}

\author{
Concretes with recycled aggregates of \\ construction and demolition waste and \\ mineral additions: a bibliographic analysis
}

\author{
Camila Lacerda Gomes ${ }^{1}$, Flávia Spitale Jacques Poggiali ${ }^{1}$, \\ Rogério Cabral de Azevedo ${ }^{1}$
}

\footnotetext{
${ }^{1}$ Programa de Pós-Graduação em Engenharia Civil - PPGEC - Centro Federal de Educação Tecnológica de Minas Gerais - Av. Amazonas, 7675, Nova Gameleira, CEP: 30510-000, Belo Horizonte, Minas Gerais, Brasil. e-mail: camilalgomes@hotmail.com, flaviaspitale@gmail.com, rogerio@civil.cefetmg.br
}

\section{RESUMO}

Os impactos ambientais causados pela produção do concreto precisam ser reduzidos para torná-lo um material mais sustentável. O uso de agregados reciclados em substituição aos agregados naturais e das adições minerais em substituição parcial ao cimento são alternativas que podem tornar o concreto um material com menor carga de poluentes associada à sua produção. Com o uso da metodologia do ProKnow-C (Knowledge Development Process - Construtivist), foi realizada uma seleção e análise de artigos com o objetivo de avaliar o cenário de pesquisas recentes sobre concretos com agregados reciclados de resíduos de construção e demolição (RCD) combinados com diversos tipos de adições minerais. Por meio da metodologia ProKnow-C obteve-se um portfólio bibliográfico composto por onze artigos. Os artigos do portfólio foram analisados para avaliar quais adições minerais e as proporções que foram utilizadas e sua eficiência para melhorar o desempenho mecânico e a durabilidade do concreto com agregados reciclados, em substituição total ou parcial ao agregado natural, tanto fino quanto graúdo. A pesquisa mostrou que agregados reciclados de RCD podem ser utilizados em altas porcentagens de substituição aos agregados naturais quando combinados com o uso de algum tipo de adição mineral. Entre as adições utilizadas nas pesquisas avaliadas estão: a sílica ativa, cinza volante e escória granulada de alto forno e cada tipo de adição utilizada apresenta um percentual ideal para ser utilizado em substituição parcial ao cimento.

Palavras-chave: Adição mineral, agregado reciclado, resíduo de construção e demolição, Proknow-C.

\section{ABSTRACT}

The environmental impact caused by the production of concrete must be reduced to make it a more sustainable material. The use of recycled aggregates in place of natural aggregates and mineral admixture in partial replacement to cement are alternatives that can make concrete a material with lower load of pollutants associated with your production. Using the methodology of ProKnow-C (Knowledge Development Process Construtivist), was performed a selection and analysis of articles to assess the scene of recent research on concrete with recycled aggregate from construction waste and demolition (CDW) combined with different kinds of mineral admixture. Through the ProKnow-C methodology was obtained a bibliographic portfolio composed of eleven papers. The portfolio items were analyzed to assess which mineral additions and ratio that were used and your efficiency to improve mechanical performance and durability of the recycled aggregate concrete in total or partial replacement to the natural aggregate, both fine and coarse. Research has shown that recycled aggregates of CDW can be used at higher replacement ratio for natural aggregates when combined with the use of some kind of mineral admixture. Among the additions used in research evaluated are silica fume, fly ash and granulated blast furnace slag and each type of admixture used presents an ideal ratio to be used in partial replacement for the cement.

Keywords: Mineral admixture, recycled aggregate, construction and demolition waste, ProKnow-C. 


\section{INTRODUÇÃO}

A produção de concreto é um dos maiores causadores de impactos ambientais e emissões de $\mathrm{CO}_{2}$. Reduzir o consumo de agregados naturais e cimento é um importante fator para minimizar impactos e preservar os recursos naturais [1] [2]. Em algumas partes do mundo a oferta de agregados é limitada, sendo importante o uso de agregados reciclados como fontes alternativas para suprir a demanda [3].

Os agregados representam grande parte do volume total do concreto, sendo um componente essencial para sua produção e de grande importância nas propriedades finais no estado fresco e endurecido [2] [4].

Reutilizar e reciclar os resíduos gerados pela construção civil é uma importante contribuição para a preservação do meio ambiente, pois aumenta o ciclo de vida dos materiais [5]. A reutilização dos resíduos como agregados na produção do concreto preserva as fontes naturais de agregados e também reduz a necessidade de aterros ao reduzir a quantidade de descartes [2] [5] [6].

Os agregados reciclados de concreto (ARC) apresentam argamassa residual aderida à sua superfície que o torna mais poroso, com menor densidade específica, maior capacidade de absorção de água, apresentando propriedades físicas e mecânicas inferiores quando comparado aos agregados naturais (AN) [2] [7].

A utilização de adições minerais como substituição parcial ao cimento também é um importante fator para reduzir a emissão de $\mathrm{CO}_{2}$ do concreto [2], uma vez que o uso destas adições em concreto, contendo agregados reciclados, melhoram a resistência à compressão e a durabilidade do mesmo. Da mesma forma, sua utilização consome menos energia e libera menos $\mathrm{CO}_{2}$ que a produção de cimento e minimiza o descarte de materiais em aterros [1] [4] [8].

Diante ao exposto, o presente estudo tem o objetivo de avaliar o cenário atual de pesquisas sobre o uso de ARC combinados com adições minerais em misturas de concreto. As informações obtidas sobre o comportamento mecânico e de durabilidade dessas misturas são importantes para identificar quais adições apresentam os melhores resultados quando utilizadas de maneira conjunta com o ARC e em quais proporções de uso essas melhorias ocorrem. A partir dessas análises, espera-se identificar as melhores proporções de uso desses materiais e as possíveis lacunas de pesquisa sobre o tema que possam fundamentar futuras pesquisas com esses materiais a fim de gerar novas contribuições ao meio acadêmico. Para tal, utilizou-se do ProKnowC para a seleção e análise dos artigos.

\section{MATERIAIS E MÉTODOS}

\subsection{Enquadramento metodológico da pesquisa}

A pesquisa apresentada neste artigo é de natureza aplicada, uma vez que se destina à obtenção de informações sobre o uso de resíduos de construção e demolição e adição de cinza de casca de arroz em concretos para reduzir os impactos ambientais, possuindo assim uma aplicação prática e dirigida à solução de problemas específicos e de interesse local [9] [10].

Com relação à forma de abordagem do problema, a pesquisa se enquadra tanto na classificação qualitativa quanto na quantitativa feita por SILVA e MENEZES [9] e KAUARK et al. [10]. As primeiras etapas da pesquisa são caracterizadas por uma análise qualitativa dos títulos, resumos e textos dos artigos, realizados pelo pesquisador, e, a seguir, análises quantitativas para determinação da relevância do portfólio selecionado.

De acordo com os objetivos dessa pesquisa, ela pode ser classificada como exploratória uma vez que busca avaliar o estado da arte do tema por meio de um levantamento bibliográfico. Esse tipo de pesquisa objetiva a maior familiaridade com o problema [9] [10].

A pesquisa foi elaborada a partir do levantamento bibliográfico de artigos publicados em periódicos das bases de busca selecionadas.

\subsection{Seleção do portfólio bibliográfico (ProKnow-C)}

O método ProKnow-C foi escolhido por sua aceitação acadêmica e capacidade de fornecer meios, não apenas para a seleção de um portfólio relevante, como também para a análise das informações contidas no portfólio, segundo a visão do pesquisador [11] [12] [13] [14] [15].

Nesta pesquisa, o ProKnow-C foi utilizado para a seleção e análise de referências bibliográficas sobre o uso de adições minerais em concretos fabricados com agregados reciclados de resíduos de construção de demolição. Foram definidos três grupos de palavras chaves que foram combinadas para realizar a pesquisa. As palavras chaves estão apresentadas na Tabela 1. 
Tabela 1: Palavras-chave de pesquisa.

\begin{tabular}{l|l|l}
\hline GRUPO 1 & GRUPO 2 & GRUPO 3 \\
\hline \multirow{2}{*}{ RECYCLED AGGREGATE } & $\begin{array}{l}\text { MINERAL ADMIXTURE } \\
\text { RICE HUSK ASH }\end{array}$ & $\begin{array}{l}\text { STRENGTH } \\
\text { DURABILITY } \\
\text { CONCRETE }\end{array}$ \\
\hline
\end{tabular}

Foram utilizadas inicialmente doze bases de busca, todas com acesso disponível através do portal de periódicos da CAPES [16]. As combinações de palavras chave foram pesquisadas em todas as bases, fornecendo um total de 5557 artigos. Das doze bases inicialmente pesquisadas, foram selecionadas quatro bases para a pesquisa, uma vez que quatro destas continham $80 \%$ do total de artigos. Além destas, foram selecionadas mais duas bases por seu conteúdo específico, relacionado ao concreto. Com isto, obteve-se um total de 4871 artigos. As bases com as respectivas quantidades de artigos obtidos como resultados das buscas são apresentadas na Figura 1.

\begin{tabular}{|c|c|}
\hline Bases de busca & Quantidade de artigos \\
\hline ScienceDirect & 2491 \\
\hline Technology collection & 1045 \\
\hline Springerlink & 460 \\
\hline Maney Publishing & 458 \\
\hline Wiley & 309 \\
\hline ASCE & 221 \\
\hline ICE & 196 \\
\hline Compendex & 155 \\
\hline Web of Science & 127 \\
\hline Scopus & 79 \\
\hline AIP Scitation & 16 \\
\hline PNAS & 0 \\
\hline
\end{tabular}

Figura 1: Bases de busca de periódicos.

Os 4871 artigos passaram pelas etapas de seleção, onde foram avaliados para eliminar artigos duplicados, verificar alinhamento do título ao tema da pesquisa, relevância acadêmica, alinhamento do resumo ao tema da pesquisa e, por fim, o alinhamento integral do texto ao tema. Ao final do processo, os artigos selecionados foram denominados de "portfólio bibliográfico".

A análise individual dos artigos foi realizada para obter os pontos de pesquisa considerados importantes para a identificação das melhores proporções de uso do ARC com adições minerais e das lacunas de pesquisa. Dessa forma, foram avaliadas as proporções de mistura utilizadas, os resultados dos ensaios mecânicos e de durabilidade apresentados para cada material e as justificativas dos pesquisadores para os resultados obtidos. A partir dessas informações, pode-se propor novas misturas e ensaios que forneçam resultados ainda não avaliados em pesquisas.

\section{RESULTADOS}

\subsection{Análise do portfólio bibliográfico}

O primeiro passo da seleção dos artigos foi a eliminação de artigos duplicados (oriundos de diferentes bases ou de diferentes combinações de palavras chave). Após esta filtragem, o total de artigos foi reduzido de 4871 para 1615. A seguir, os artigos foram avaliados em relação ao alinhamento de seu título com o tema desta pesquisa, resultando em 42 artigos com título totalmente alinhado com o tema da pesquisa.

O próximo passo do processo de seleção foi a filtragem pela relevância acadêmica, dada pela quantidade de citações que cada artigo obteve até a data de realização deste processo. Neste passo, selecionou-se os artigos cuja soma das citações corresponda a $85 \%$ do total de citações dos 42 artigos, o que resultou em 13 artigos. A seguir, os resumos dos 13 artigos foram avaliados e 10 artigos foram selecionados (título e resumo 
alinhado com o tema da pesquisa e relevância acadêmica).

No penúltimo passo do processo, buscou-se identificar artigos recentes que possuem poucas citações e artigos dos autores selecionados anteriormente. $\mathrm{O}$ resumo destes artigos foi avaliado e sete foram adicionados aos já selecionados anteriormente. O portfólio passou a contar, então, com 17 artigos.

Como passo final do processo de seleção, os artigos foram analisados na íntegra e 11 foram selecionados como totalmente alinhados com o tema e propósito da pesquisa, os quais estão apresentados na Tabela 2.

Tabela 2: Artigos selecionados.

\begin{tabular}{|c|c|c|c|}
\hline REFERÊNCIA & AUTORES & TíTULO & ANO \\
\hline [1] & BERNDT, M. L. & $\begin{array}{l}\text { Properties of sustainable concrete containing fly } \\
\text { ash, slag and recycled concrete aggregate }\end{array}$ & 2009 \\
\hline [17] & CORINALDESI, V.; MORICONI, G. & $\begin{array}{l}\text { Influence of mineral additions on the perfor- } \\
\text { mance of } 100 \% \text { recycled aggregate concrete }\end{array}$ & 2009 \\
\hline [5] & $\begin{array}{l}\text { GONZÁLEZ-FONTEBOA, B.; MAR- } \\
\text { TÍNEZ-ABELLA, F. }\end{array}$ & $\begin{array}{l}\text { Concretes with aggregates from demolition } \\
\text { waste and silica fume. Materials and mechani- } \\
\text { cal properties }\end{array}$ & 2008 \\
\hline [7] & $\begin{array}{l}\text { KAPOOR, K.; SINGH, S. P. P.; } \\
\text { SINGH, B. }\end{array}$ & $\begin{array}{l}\text { Durability of self-compacting concrete made } \\
\text { with Recycled Concrete Aggregates and mineral } \\
\text { admixtures }\end{array}$ & 2016 \\
\hline [3] & $\begin{array}{l}\text { KOU, S. C.; POON, C. S.; AGRELA, } \\
\text { F. }\end{array}$ & $\begin{array}{l}\text { Comparisons of natural and recycled aggregate } \\
\text { concretes prepared with the addition of different } \\
\text { mineral admixtures }\end{array}$ & 2011 \\
\hline [18] & KOU, S. C.; POON, C. S. & $\begin{array}{l}\text { Enhancing the durability properties of concrete } \\
\text { prepared with coarse recycled aggregate }\end{array}$ & 2012 \\
\hline [2] & $\begin{array}{l}\text { LIMBACHIYA, M.; MEDDAH, M. S.; } \\
\text { OUCHAGOUR, Y. }\end{array}$ & $\begin{array}{l}\text { Use of recycled concrete aggregate in fly-ash } \\
\text { concrete }\end{array}$ & 2012 \\
\hline [19] & $\begin{array}{l}\text { SOMNA, R.; JATURAPITAKKUL, C.; } \\
\text { AMDE, A. M. }\end{array}$ & $\begin{array}{l}\text { Effect of ground fly ash and ground bagasse ash } \\
\text { on the durability of recycled aggregate concrete }\end{array}$ & 2012 \\
\hline [6] & $\begin{array}{l}\text { SOMNA, R.; JATURAPITAKKUL, C.; } \\
\text { CHALEE, W.; RATTANACHU, P. }\end{array}$ & $\begin{array}{l}\text { Effect of the Water to Binder Ratio and Ground } \\
\text { Fly Ash on Properties of Recycled Aggregate } \\
\text { Concrete }\end{array}$ & 2012 \\
\hline [8] & $\begin{array}{l}\text { TANGCHIRAPAT, W.; BURA- } \\
\text { NASING, R.; JATURAPITAKKUL, } \\
\text { C.; CHINDAPRASIRT, P. }\end{array}$ & $\begin{array}{l}\text { Influence of rice husk-bark ash on mechanical } \\
\text { properties of concrete containing high amount } \\
\text { of recycled aggregates }\end{array}$ & 2008 \\
\hline [4] & $\begin{array}{l}\text { TANGCHIRAPAT, W.; RAT- } \\
\text { TANASHOTINUNT, C.; BURA- } \\
\text { NASING, R.; JATURAPITAKKUL, C. }\end{array}$ & $\begin{array}{l}\text { Influence of Fly Ash on Slump Loss and Strength } \\
\text { of Concrete Fully Incorporating Recycled Con- } \\
\text { crete Aggregates }\end{array}$ & 2013 \\
\hline
\end{tabular}

A análise bibliométrica dos artigos selecionados tem o objetivo de evidenciar a relevância destes, dos periódicos em que foram publicados e dos seus autores.

Os artigos selecionados para o portfólio foram publicados em quatro periódicos, sendo o Construction and Building Materials o de maior relevância, dada pela contagem de artigos no portfólio. Também foi avaliada a representatividade dos quatro periódicos dentro das referências. A Figura 2 apresenta a análise de relevância dos periódicos dentro do portfólio, dada pela contagem dos artigos selecionados e pela contagem desses nas referências do portfólio. A classificação dos periódicos de acordo com o Qualis-Periódicos [20] é apresentada junto ao seu título na Figura 2. 


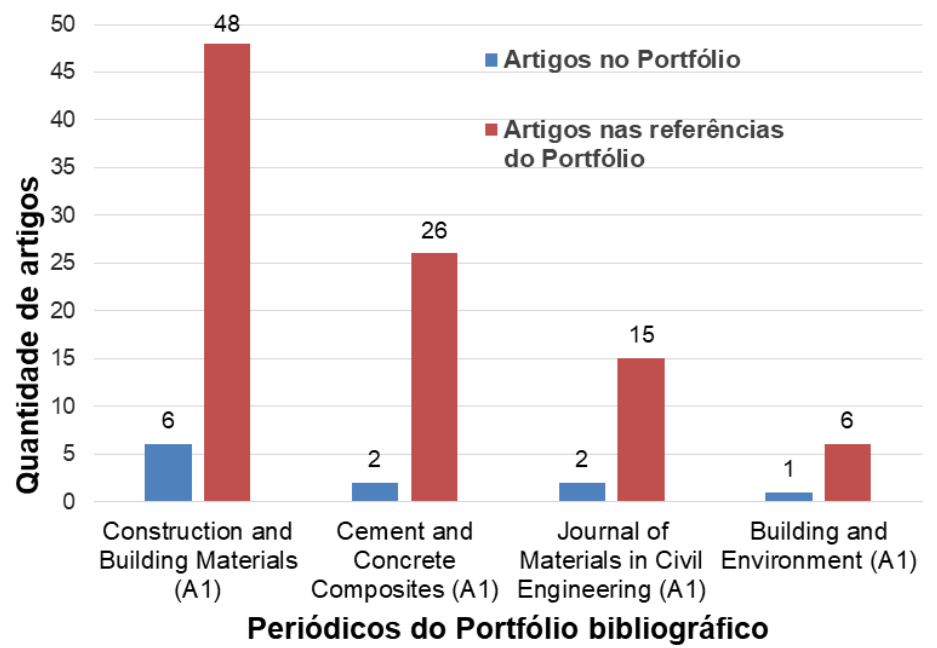

Figura 2: Relevância dos periódicos.

A Figura 3 apresenta as publicações com suas respectivas citações, obtidas por contagem no Google Acadêmico em 11 de outubro de 2017, e nas referências dos artigos do portfólio. Nas citações das referências, o artigo "Properties of sustainable concrete containing fly ash, slag and recycled concrete aggregate" apresenta maior destaque, tendo sido citado em 4 das publicações selecionadas. Pelas citações do Google Acadêmico, dois artigos recebem maior destaque, sendo eles "Properties of sustainable concrete containing fly ash, slag and recycled concrete aggregate" e "Influence of mineral additions on the performance of $100 \%$ recycled aggregate concrete".

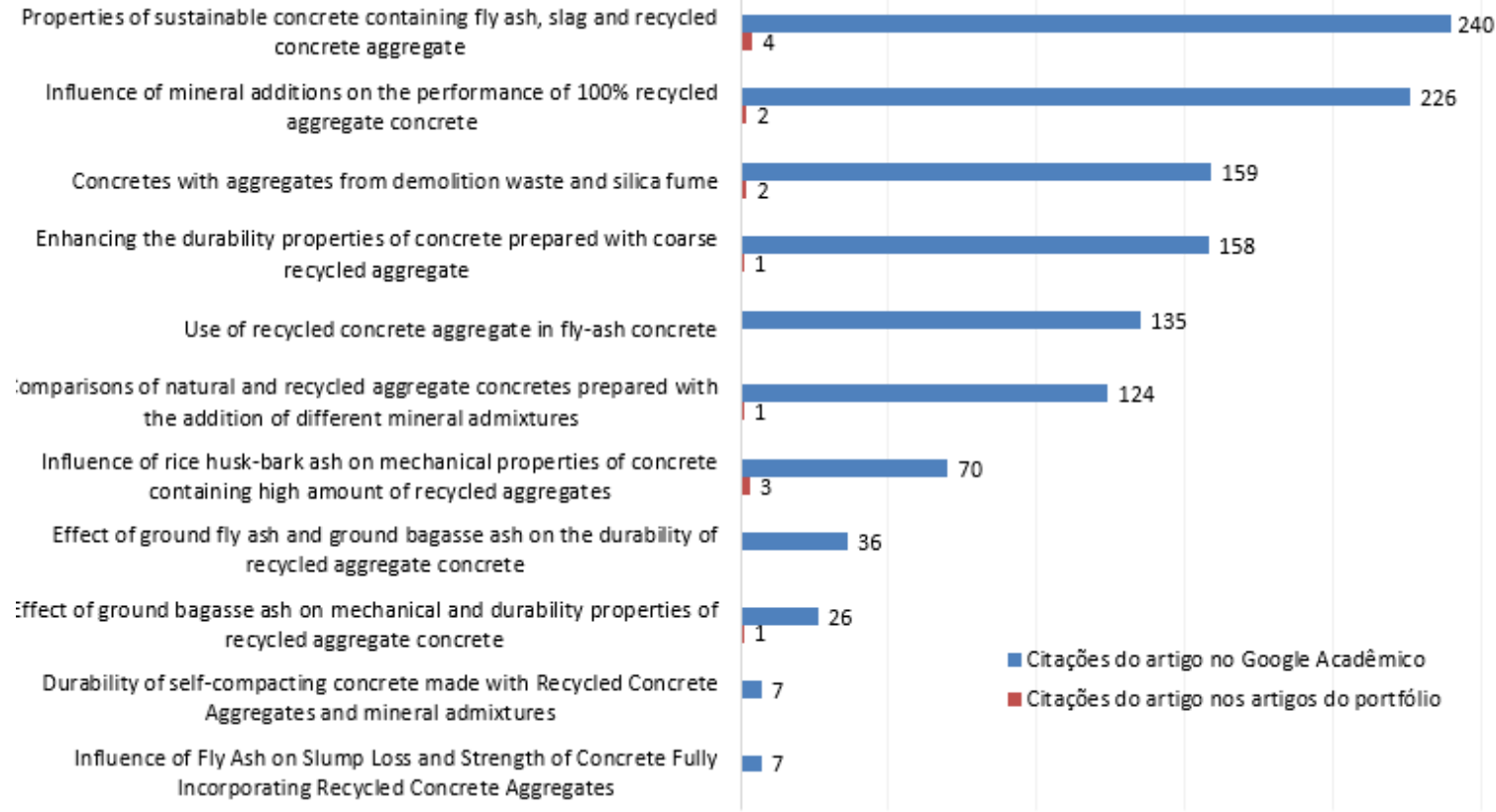

Figura 3: Relevância dos artigos.

A Figura 4 apresenta a análise de relevância dos autores dos artigos do portfólio. C. Jaturapitakkul foi o autor com mais artigos, tendo participado de 4 dos 11 artigos. Os autores que se destacaram C. Jaturapitakkul e C. S. Poon, tendo cada um deles aparecido 23 vezes nas referências. 


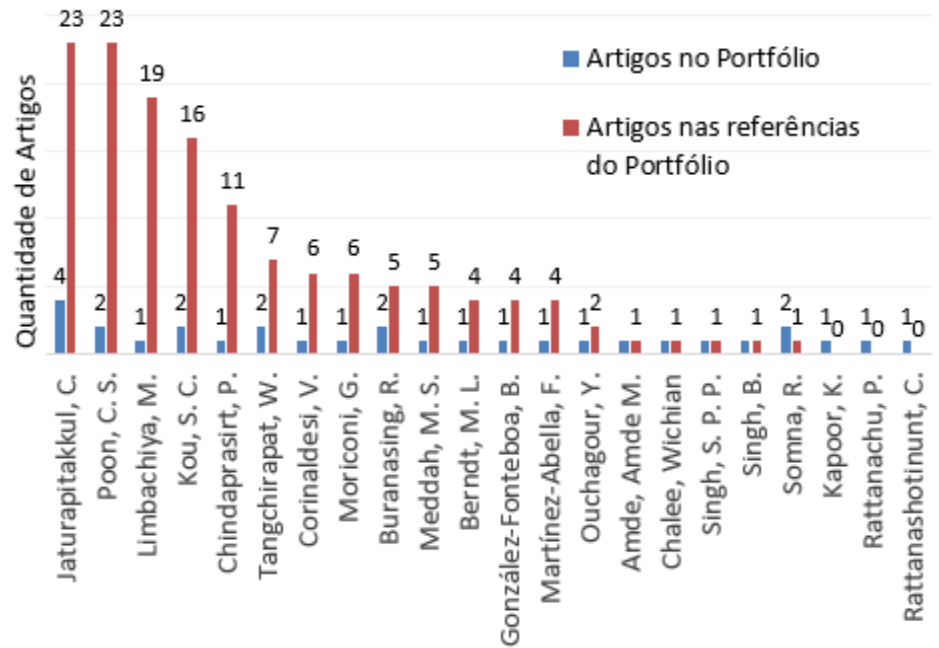

Figura 4: Relevância dos atores.

\section{DISCUSSÃO}

Os artigos avaliados abordam diferentes percentuais de substituição dos agregados naturais por reciclados e de cimento por diferentes adições minerais. A Tabela 3 apresenta quais agregados e adições são utilizados nas substituições e as proporções utilizadas em cada pesquisa.

Tabela 3: Proporções de misturas.

\begin{tabular}{|c|c|c|c|c|}
\hline AUTOR & $\begin{array}{c}\text { PERCENTUAL } \\
\text { DE ARG }\end{array}$ & $\begin{array}{l}\text { PERCENTUAL } \\
\text { DE ARF }\end{array}$ & $\begin{array}{l}\text { TIPO DE ADIÇÃO } \\
\text { MINERAL }\end{array}$ & $\begin{array}{l}\text { PERCENTUAL DE } \\
\text { ADIÇÃO MINERAL }\end{array}$ \\
\hline $\begin{array}{l}\text { GONZÁLEZ-FONTEBOA E } \\
\text { MARTÍNEZ-ABELLA [5] }\end{array}$ & $50 \%$ & - & SA & $8 \%$ \\
\hline TANGCHIRAPAT et al. [8] & $100 \%$ & 0,50 e $100 \%$ & $\mathrm{CCA} / \mathrm{CE}$ & 20,35 e $50 \%$ \\
\hline BERNDT [1] & $100 \%$ & - & EGAF & 50 e $70 \%$ \\
\hline CORINALDESI E MORICONI [17] & $100 \%$ & $100 \%$ & $\begin{array}{l}\mathrm{CV} \\
\mathrm{SA}\end{array}$ & $\begin{array}{l}30 \% \\
15 \%\end{array}$ \\
\hline KOU et al. [3] & 50 e $100 \%$ & - & \begin{tabular}{|l|} 
SA \\
MC \\
CV \\
EGAF \\
\end{tabular} & $\begin{array}{l}10 \% \\
15 \% \\
35 \% \\
55 \% \\
\end{array}$ \\
\hline LIMBACHIYA et al. [2] & 30,50 e $100 \%$ & - & $\mathrm{CV}$ & $30 \%$ \\
\hline SOMNA et al. [19] & $100 \%$ & - & $\begin{array}{l}\mathrm{CV} \\
\mathrm{CBC}\end{array}$ & 20,35 e $50 \%$ \\
\hline KOU e POON [18] & 20,50 e $100 \%$ & - & $\mathrm{CV}$ & $25 \%$ \\
\hline SOMNA et al. [6] & $100 \%$ & - & $\mathrm{CV}$ & 20,35 e $50 \%$ \\
\hline TANGCHIRAPAT et al. [4] & $100 \%$ & 0 e $100 \%$ & $\mathrm{CV}$ & 20,35 e $50 \%$ \\
\hline KAPOOR et al. [7] & 50 e $100 \%$ & - & $\begin{array}{l}\mathrm{CV} \\
\mathrm{SA} \\
\mathrm{MC}\end{array}$ & 10,20 e $30 \%$ \\
\hline
\end{tabular}

ARG - Agregado reciclado graúdo; ARF - Agregado reciclado fino; SA - Sílica ativa; CCA/CE - Cinza de casca de arroz e casca de eucalipto; EGAF - Escória granulada de alto forno; CV - Cinza volante; MC - Metacaulim; CBC - Cinza de bagaço de cana.

A Tabela 4 apresenta os melhores resultados de resistência à compressão, módulo de elasticidade e resistência à tração de cada estudo. Os valores indicados se referem à diferença percentual entre a mistura com ARC e adição que apresentou os melhores resultados com relação à mistura controle, sendo que os valores positivos representam misturas que obtiveram resultados superiores à mistura controle e negativos para resultados inferiores. 
Tabela 4: Resultados das propriedades mecânicas.

\begin{tabular}{|c|c|c|c|c|}
\hline AUTOR & PROPORÇÃO DE MISTURA & $\begin{array}{l}\text { RESISTÊNCIA À } \\
\text { COMPRESSÃO }\end{array}$ & $\begin{array}{l}\text { MÓDULO DE } \\
\text { ELASTICIDADE }\end{array}$ & $\begin{array}{l}\text { RESISTÊNCIA } \\
\text { À TRAÇÃO }\end{array}$ \\
\hline $\begin{array}{l}\text { GONZÁLEZ-FONTEBOA E } \\
\text { MARTÍNEZ-ABELLA [5] }\end{array}$ & $50 \% \mathrm{ARG}+8 \% \mathrm{SA}$ & $+8,29 \%$ & $-15,19$ & $+6,78 \%$ \\
\hline TANGCHIRAPAT et al. [8] & $100 \% \mathrm{ARG}+20 \% \mathrm{CCA} / \mathrm{CE}$ & $+7,44 \%$ & $-4,58$ & $-7,32 \%$ \\
\hline BERNDT [1] & $100 \%$ ARG +50\%EGAF & $+15,30$ & $+0,42 \%$ & $+17,03 \%$ \\
\hline $\begin{array}{l}\text { CORINALDESI E MORICONI } \\
{[17]}\end{array}$ & $\begin{array}{l}\begin{array}{l}100 \% \mathrm{ARG}+100 \% \mathrm{ARF} \\
+30 \% \mathrm{CV}\end{array} \\
\end{array}$ & $+0,59 \%$ & $-31,72 \%$ & $-10,13$ \\
\hline KOU et al. [3] & $50 \% \mathrm{ARG}+15 \% \mathrm{MC}$ & $-0,70 \%$ & NA & $-6,65 \%$ \\
\hline LIMBACHIYA et al. [2] & $100 \% \mathrm{ARG}+30 \% \mathrm{CV}$ & $+18,85 \%$ & $-36,36 \%$ & NA \\
\hline SOMNA et al. [19] & $100 \% \mathrm{ARG}+20 \% \mathrm{CV}$ & $-16,67 \%$ & NA & NA \\
\hline KOU e POON [18] & $50 \% \mathrm{ARG}+25 \% \mathrm{CV}$ & $-14,20 \%$ & NA & NA \\
\hline SOMNA et al. [6] & $100 \% \mathrm{ARG}+20 \mathrm{CV}$ & $-3,83 \%$ & $-16,00 \%$ & NA \\
\hline TANGCHIRAPAT et al. [4] & $100 \% \mathrm{ARG}+35 \% \mathrm{CV}$ & $+6,17 \%$ & $-7,16 \%$ & $-8,74 \%$ \\
\hline KAPOOR et al. [7] & $50 \% \mathrm{ARG}+30 \mathrm{CV}$ & $-4,45 \%$ & NA & NA \\
\hline
\end{tabular}

(+) Resultado superior à mistura controle

(-) Resultado inferior à mistura controle

NA - Não avaliado no estudo

A partir da análise dos resultados de ensaios apresentados em cada artigo, é possível identificar que cada adição apresenta proporções ideais de substituição quando utilizadas em conjunto com o ARC. Como cada estudo utiliza diferentes propostas de incorporação, tanto dos agregados quanto das adições minerais, este trabalho apresenta os resultados de cada um deles individualmente.

\subsection{Estudo de GONZÁLEZ-FONTEBOA E MARTíNEZ-ABELLA [5]}

Os pesquisadores utilizaram ARC e sílica ativa (SA) para confeccionar o concreto e realizar testes de resistência à compressão, resistência à tração, módulo de elasticidade e absorção de água. Os ensaios foram realizados aos 7, 28 e 115 dias.

Nos testes de absorção de água aos 115 dias, as misturas contendo agregados reciclados e/ou sílica ativa apresentaram proporções de absorção maiores que a mistura de concreto convencional (CC).

Os resultados de resistência à compressão aos 28 dias apresentaram valores mais baixos para a mistura contendo ARC quando comparado à mistura CC. Entretanto, a mistura contendo ARC e adição de SA apresentou resultado mais alto que o CC.

Para a resistência à ruptura por tração, os autores concluíram que a adição de sílica ativa e dos agregados reciclados não provocaram impactos na resistência.

A mistura contendo ARC e SA apresentou resultado inferior para o módulo de elasticidade, correspondendo cerca de $85 \%$ do valor obtido para a mistura CC.

\subsection{Estudo de TANGCHIRAPAT et al. [8]}

Os autores utilizaram substituição total de agregados graúdos e parciais de agregados finos por ARC. As cinzas de casca de arroz e eucalipto (CCA/CE) foram utilizadas para substituir parcialmente o cimento. Foram realizados testes de resistência à compressão, resistência à tração e módulo de elasticidade, aos 7, 28 e 90 dias.

Os autores observaram que o uso de ARC reduziu a resistência à compressão do concreto, sendo os maiores valores de redução obtidos quando da substituição dos agregados graúdos e finos. A incorporação das cinzas, em percentuais de $20 \%$ e $35 \%$ de substituição, juntamente com o agregado reciclado graúdo apresentaram maior resistência que a mistura $\mathrm{CC}$.

Os valores medidos para o módulo de elasticidade de todas as misturas com ARC foram menores que o do concreto controle. Misturas utilizando somente ARC apresentaram redução de $24 \%$ no resultado do módulo de elasticidade, enquanto as misturas com substituição apenas do agregado graúdo natural pelo ARC obtiveram resultados $11 \%$ menores que a mistura $\mathrm{CC}$.

A resistência à ruptura por tração não indicou diferença significativa entre os resultados obtidos para 
as diversas misturas.

\subsection{Estudo de BERNDT [1]}

$\mathrm{O}$ autor realizou misturas com substituição total do agregado graúdo por ARC e substituições parciais de cimento por $50 \%$ e $70 \%$ de escória granulada de alto-forno (EGAF). Foram realizados testes de resistência à compressão, resistência à tração, módulo de elasticidade, coeficiente de permeabilidade e coeficiente de difusão de cloretos.

$\mathrm{O}$ autor avaliou que a resistência à compressão aos 28 dias dos concretos contendo ARC e EGAF apresentaram valores melhores que o concreto controle: 43,7 MPa para 50\% de escória, 41,2 MPa para 70\% de escória e 37,9 MPa para o concreto controle.

O módulo de elasticidade das misturas contendo ARC e EGAF foram menores que o obtido para o concreto controle.

As misturas com ARC e EGAF apresentaram valor de resistência à tração equivalente ou superior ao obtido para a mistura controle.

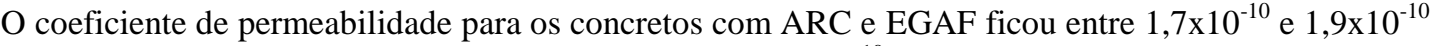
$\mathrm{cm} / \mathrm{s}$, apresentando valores maiores que o concreto controle, $1,0 \times 10^{-10}$, porém ainda ficaram abaixo do limite para que o concreto seja considerado durável, que é de $3,0 \times 10^{-10} \mathrm{~cm} / \mathrm{s}$, segundo a ASTM D 5084, norma seguida pelos autores para realização do ensaio.

O coeficiente de difusão de íons cloreto apresentou resultados mais favoráveis para os concretos com o uso de ARC e EGAF quando comparados ao concreto controle.

\subsection{Estudo de CORINALDESI E MORICONI [17]}

Os autores estudaram concretos com substituição total dos agregados naturais finos e graúdos por ARC e também substituições do agregado reciclado fino por 30\% de cinzas volantes (CV) ou 15\% de sílica ativa (SA).

Em sua primeira etapa de pesquisa, os autores obtiveram concretos com diferentes classes de resistência à compressão para as misturas realizadas: classe de resistência de 25 MPa para o concreto com ARC, 30 MPa para o concreto controle e o concreto contendo ARC e CV, e 40 MPa para mistura contendo ARC e SA.

O resultado do módulo de elasticidade secante para a mistura contendo SA apresentou valor 10,9\% superior ao concreto controle, enquanto a mistura com CV apresentou redução de 9,9\%. Para o módulo tangente, a mistura com SA apresenta acréscimo de $9,8 \%$ e a mistura com CV apresenta redução de $14,3 \%$ quando comparados ao resultado do CC.

Para o módulo de elasticidade dinâmico, todas as misturas apresentaram redução no resultado com relação à mistura controle, sendo a redução de 19,9\% e 31,5\% para as misturas com SA e CV, respectivamente.

Os autores destacam que os módulos estáticos dependem da resistência à compressão, já o módulo dinâmico tem como principal influência o tipo de agregado utilizado.

\subsection{Estudo de KOU et al. [3]}

Os autores estudaram concretos com agregados reciclados graúdos (ARG) em substituições de 50\% e 100\% aos agregados naturais e adições minerais como substituição parcial ao cimento nas proporções de $10 \%$ de sílica ativa (SA), $15 \%$ de metacaulim (MC), 35\% de cinzas volantes (CV) ou 55\% de escória granulada de alto-forno (EGAF).

Nos testes de resistência à compressão aos 28 dias, as misturas contendo agregados reciclados e adições de $\mathrm{SA}$ ou $\mathrm{MC}$ apresentaram resultados próximos à mistura de referência. Já as misturas contendo agregados reciclados e adições de CV ou EGAF obtiveram resultados de resistência à compressão significativamente mais baixos.

Aos 28 dias, os testes de resistência à tração mostraram que as misturas com agregados reciclados e adições de SA ou MC apresentaram valores próximos à referência, já aos 90 dias, as misturas apresentaram valores superiores à referência. As misturas com agregados reciclados e adições de CV ou EGAF apresentaram uma redução considerável da resistência à tração em relação à referência em todas as idades de teste.

As misturas contendo agregados reciclados e adições minerais apresentaram melhores resultados nos testes de penetração de íons cloreto aos 28 e 90 dias, onde os autores observaram que as adições melhoram a impermeabilidade do concreto melhorando a durabilidade. 


\subsection{Estudo de LIMBACHIYA et al. [2]}

Os autores avaliaram concretos com resistência de $20 \mathrm{MPa}, 30 \mathrm{MPa}$ e $35 \mathrm{MPa}$ fabricados com substituição dos agregados graúdos naturais por ARC nas proporções de 30\%, 50\% e 100\%, e utilizando CV para substituição de $30 \%$ do cimento nas misturas.

Os autores observaram que a resistência à compressão para as misturas de classe $\mathrm{C} 20$ e C30 tende a reduzir com o aumento da substituição dos agregados naturais. O uso da $\mathrm{CV}$ proporcionou valores de resistência à compressão maiores que a mistura de referência, mesmo para a substituição total dos agregados. Já as misturas de classe C35 apresentaram uma tendência contrária, atribuída pelos autores aos parâmetros intrínsecos do agregado reciclado que podem influenciar as propriedades do concreto.

Foi observado no estudo que a retração por secagem aumentou proporcionalmente à substituição dos agregados naturais. $\mathrm{O}$ uso da $\mathrm{CV}$ reduziu a retração por secagem dos concretos com agregados reciclados quando comparado à mistura utilizando apenas cimento Portland. As misturas com agregados reciclados, mesmo quando combinadas com $\mathrm{CV}$, apresentaram maior retração que a mistura de referência.

Nas misturas com $100 \%$ de agregados reciclados os autores não observaram melhora na resistência ao ataque por sulfatos com a incorporação das cinzas, já as misturas com porcentagens menores apresentaram melhorias com o uso das cinzas volantes.

A resistência à carbonatação e à penetração de íons cloretos diminuiu com o aumento de substituição dos agregados naturais. $\mathrm{O}$ uso da $\mathrm{CV}$ não proporcionou melhor significativa no desempenho das misturas.

\subsection{Estudo de SOMNA et al. [19]}

Os autores utilizaram agregados reciclados feitos a partir do esmagamento de moldes de concreto submetidos à testes de compressão, com resistência entre 25 e $40 \mathrm{MPa}$, para substituir totalmente os agregados graúdos, e substituições parciais do cimento por $\mathrm{CV}$ e cinzas de bagaço de cana-de-açúcar (CBC), em 20\%, 35\% e 50\% em peso do aglomerante.

Nos testes de resistência à compressão foi observado que a substituição dos agregados junto com a utilização de $35 \%$ ou $50 \%$ de cinzas, tanto volantes quanto de bagaço de cana, produziram concretos com resistência muito inferior à mistura de referência. Para as substituições de agregados e $20 \%$ de cinzas, as misturas apresentaram valores cerca de $10 \%$ menores que o concreto de referência, porém próximos à mistura contendo apenas agregados reciclados, sem as adições minerais.

$\mathrm{O}$ coeficiente de permeabilidade à água do concreto nas misturas contendo ARC e cinzas apresentou valores menores que a mistura de referência, sendo essa redução atribuída pelos autores ao preenchimento dos poros na matriz do concreto, que se torna mais denso com o uso das adições minerais.

Nos testes de penetração de íons cloreto, as misturas contendo ARC e cinzas apresentaram melhor desempenho que a mistura de referência, tendo reduzido consideravelmente a profundidade de penetração nos testes realizados aos 6,12 e 18 meses.

Nos testes de expansão do concreto realizados com ataque de sulfatos, as misturas contendo as cinzas melhoraram o desempenho do concreto com ARC, porém quando foi utilizado $35 \%$ e $50 \%$ de substituição do cimento foi observado danos na superfície do concreto devido à queda da resistência à compressão.

\subsection{Estudo de KOU e POON [18]}

Os autores utilizaram ARC para substituir 20\%,50\% e 100\% dos agregados graúdos. As misturas também utilizaram CV em $25 \%$ e $35 \%$ como substituição ou adição ao cimento, em peso. Foram realizados testes de resistência à compressão, penetração de íons cloreto, absorção de água por capilaridade e profundidade de carbonatação.

As misturas que utilizaram CV como substituição ao cimento apresentaram valores mais baixos de resistência à compressão aos 28 dias para todas as proporções de substituição de agregados utilizadas; aos 90 dias, as misturas utilizando $25 \%$ de cinzas e até $50 \%$ de ARC apresentaram valores de resistência à compressão maiores que a mistura controle, as demais misturas realizadas com substituição do cimento apresentaram valores inferiores ao controle aos 90 dias.

As misturas que utilizaram $25 \%$ de cinzas como adição ao cimento e substituições de até $50 \%$ de ARC apresentaram valores de resistência à compressão aos 28 dias equivalentes ou superiores à mistura controle; aos 90 dias, todas as misturas com $25 \%$ de adição de CV apresentaram valores superiores ao concreto controle na mesma idade. As misturas que utilizaram $35 \%$ de cinzas como adição ao cimento apresentaram maiores valores de resistência à compressão aos 28 e 90 dias, chegando a acrescentar mais de $40 \%$ do valor de resis- 
tência à compressão da mistura controle.

Os testes de penetração de íons cloreto mostraram que as misturas contendo ARC e CV apresentaram maior resistência à penetração, sendo que as misturas com adição das cinzas ao cimento foram mais eficientes que as misturas com substituição parcial do cimento pelas cinzas.

Nos testes de absorção de água por capilaridade, a mistura contendo 50\% de ARC e $35 \%$ de CV como substituição ao cimento apresentaram valores equivalentes à mistura controle, as demais misturas com ARC e substituição de cimento pelas cinzas apresentaram valores mais altos de absorção. Nas misturas com adição das cinzas ao cimento, as misturas contendo $50 \%$ de ARC e $25 \%$ ou $35 \%$ de cinzas apresentaram valores de absorção de água capilar menores que o controle, as demais misturas com ARC e adição de cinzas apresentaram valores acima da mistura controle.

A profundidade de carbonatação de todas as misturas contendo ARC e cinzas apresentaram valores superiores à mistura controle.

\subsection{Estudo de SOMNA et al. [6]}

Os autores substituíram 100\% dos agregados naturais graúdos por ARC utilizando substituições de cimento por CV nas proporções de $20 \%, 35 \%$ e $50 \%$ para misturas de concreto feitas com três fatores a/c distintos: $0,45,0,55$ e 0,65 . Foram realizados testes de resistência à compressão, módulo de elasticidade e permeabilidade à água.

Os autores observaram que um baixo fator a/c e maior tempo de cura melhoram a resistência à compressão do concreto com ARC e CV de maneira eficiente.

Os autores concluíram que para o fator a/c 0,45 , a CV pode substituir o cimento em até $35 \%$ nos concretos com ARC mantendo a resistência à compressão maior que a mistura com o ARC e sem adição mineral. Para as misturas com fator a/c de 0,55 e 0,65 , a taxa de substituição do cimento pelas cinzas não deve exceder $20 \%$.

O módulo de elasticidade do concreto controle ficou entre $16 \%$ e $20 \%$ maior que as misturas com ARC, com e sem uso da adição mineral. Os autores também observaram que utilizar CV foi mais efetivo para reduzir o coeficiente de permeabilidade à água que reduzir o fator a/c das misturas.

\subsection{Estudo de TANGCHIRAPAT et al. [4]}

Os autores avaliaram o uso de cinzas volantes em concretos utilizando substituição total dos agregados graúdos por ARC e também substituição dos agregados graúdos e finos. O concreto original utilizado na confecção dos agregados reciclados apresentava resistência entre 25 e $40 \mathrm{MPa}$.

Os autores relataram que o uso dos agregados reciclados graúdos aumentava a demanda de água na mistura com relação à mistura de referência e o uso de agregados reciclados finos e graúdos requeria ainda mais água.

Foi observado nos testes de resistência à compressão que o uso da CV melhorou o desempenho dos concretos contendo grande quantidade de agregados reciclados graúdos, chegando a equiparar com os valores da mistura controle.

As misturas que utilizaram agregados reciclados finos e graúdos apresentaram valores de resistência à compressão abaixo do concreto controle, mesmo utilizando as cinzas, o melhor resultado para essa combinação foi de $85 \%$ do valor da resistência à compressão da mistura controle.

\subsection{Estudo de KAPOOR et al. [7]}

Os autores estudaram a substituição do agregado natural graúdo em diferentes proporções de ARC e substituição parcial do cimento por $\mathrm{CV}$ combinada com SA ou metacaulim (MC) em concreto auto-adensável.

Foi observado nos testes de resistência à compressão que a substituição de $50 \%$ dos agregados graúdos e o uso das adições de CV juntamente com SA ou MC obtiveram valores comparáveis ao concreto controle.

Os testes de penetração de íons cloreto mostraram que as misturas contendo as adições apresentaram melhores resultados que a mistura controle.

As misturas com as adições e 50\% de ARC mostraram melhoria de desempenho nos testes de absorção superficial inicial, já o uso de $100 \%$ dos agregados reciclados forneceu valores de absorção maiores que o concreto controle. 


\section{CONCLUSÕES}

A análise dos artigos permitiu identificar que a maior parte dos estudos envolve o uso de cinzas volantes ou sílica ativa como adição mineral. $\mathrm{O}$ uso das cinzas de casca de arroz e de bagaço de cana-de-açúcar foi relatado em apenas um artigo cada, o que dá indícios que essas adições minerais combinadas com agregados reciclados de resíduos de construção e demolição são menos exploradas em pesquisas desenvolvidas.

As proporções ideais de substituição dos agregados e do cimento irão depender das características específicas destes dois materiais, que podem variar de acordo com a fonte de obtenção dos agregados reciclados e das adições minerais a serem utilizadas.

A partir da análise dos resultados de ensaios apresentados em cada artigo, é possível identificar que cada adição apresenta proporções ideais de substituição quando utilizadas em conjunto com o ARG. Para utilização do ARC em substituição aos agregados finos, as adições não se mostraram eficientes para equiparar as propriedades das misturas propostas ao concreto controle.

A Tabela 4 apresentou os melhores resultados das propriedades mecânicas de cada estudo. A resistência à compressão obteve melhor resultado no estudo de LIMBACHIYA et al. [2], onde apresentou ganho de $18,85 \%$ em relação ao concreto controle.

Para o uso de $\mathrm{CV}$, a faixa de substituição que apresentou resultados favoráveis nos ensaios está entre $20 \%$ e $35 \%$. Esses percentuais se mostraram eficientes com uso do ARG em todas as proporções de substituição.

Misturas com 50\% de ARC em substituição aos agregados graúdos e 10\% de SA apresentaram melhores resultados nos testes de resistência à compressão e à penetração de íons cloreto em comparação ao concreto controle.

O uso de CCA/CE, em proporções de $20 \%$ e $35 \%$, e ARC, em substituição total dos agregados graúdos, resultou em misturas com resistência à compressão equivalente ou superior ao concreto controle.

Adições de EGAF em proporções entre 50\% e 70\% juntamente com o ARC mostrou resultados de resistência à compressão superiores ao concreto controle. O uso de 55\% de EGAF apresentou resistência à penetração de íons cloreto superior ao concreto controle.

Misturas contendo $15 \%$ de $\mathrm{MC}$ e ARC apresentaram resultados de resistência à compressão e à penetração de íons cloretos melhores que o concreto controle.

De maneira geral, as propriedades das misturas de concreto com ARC e as adições que não apresentaram resultados equivalentes à mistura controle acabaram apresentando melhores resultados quando comparadas ao concreto com ARC e sem adições.

Os resultados evidenciados nesta pesquisa comprovam que o uso de grandes quantidades de agregados reciclados graúdos em concretos pode ser viabilizado por meio do uso de adições minerais. $\mathrm{O}$ aproveitamento desses materiais, de acordo com a disponibilidade de cada região, pode tornar o concreto um material mais sustentável, reduzindo os impactos da extração de matéria-prima e do descarte de resíduos.

\section{AGRADECIMENTOS}

Os autores agradecem o suporte do CEFET-MG e CAPES.

\section{BIBLIOGRAFIA}

[1] BERNDT, M. L. "Properties of sustainable concrete containing fly ash, slag and recycled concrete aggregate", Construction and Building Materials, v. 23, n. 7, pp. 2606-2613, Jul. 2009.

[2] LIMBACHIYA, M., MEDDAH, M. S., OUCHAGOUR, Y. "Use of recycled concrete aggregate in flyash concrete", Construction and Building Materials, v. 27, n. 1, pp. 439-449, Feb. 2012.

[3] KOU, S. C., POON, C. S., AGRELA, F. "Comparisons of natural and recycled aggregate concretes prepared with the addition of different mineral admixtures", Cement and Concrete Composites, v. 33, n. 8, pp. 788-795, Sep. 2011.

[4] TANGCHIRAPAT, W., RATTANASHOTINUNT, C., BURANASING, R., et al., "Influence of Fly Ash on Slump Loss and Strength of Concrete Fully Incorporating Recycled Concrete Aggregates", Journal of Materials in Civil Engineering, v. 25, n. 2, pp. 243-251, Feb. 2013.

[5] GONZÁLEZ-FONTEBOA, B., MARTÍNEZ-ABELLA, F. "Concretes with aggregates from demolition waste and silica fume. Materials and mechanical properties", Building and Environment, v. 43, n. 4, pp. 429437, Apr. 2008. 
[6] SOMNA, R., JATURAPITAKKUL, C., CHALEE, W., et al., "Effect of the Water to Binder Ratio and Ground Fly Ash on Properties of Recycled Aggregate Concrete", Journal of Materials in Civil Engineering, v. 24, n. 1, pp. 16-22, Jan. 2012.

[7] KAPOOR, K., SINGH, S. P. P., SINGH, B. "Durability of self-compacting concrete made with Recycled Concrete Aggregates and mineral admixtures", Construction and Building Materials, v. 128, pp. 67-76, Dec. 2016.

[8] TANGCHIRAPAT, W., BURANASING, R., JATURAPITAKKUL, C., et al., "Influence of rice huskbark ash on mechanical properties of concrete containing high amount of recycled aggregates", Construction and Building Materials, v. 22, n. 8, pp. 1812-1819, Aug. 2008.

[9] SILVA, E. L., MENEZES, E. M., Metodologia da pesquisa e elaboração de dissertação, 4 ed, Florianópolis, UFSC, 2005.

[10] KAUARK, F., MANHÃES, F. C., MEDEIROS, C. H., Metodologia da pesquisa: guia prático, Itabuna, Via Litterarum, 2010.

[11] ROSA, F. S. D., ENSSLIN, S. R., ENSSLIN, L., et al., "Environmental disclosure management: A constructivist case", Management Decision, v. 50, n. 6, pp. 1117-1136, Jun. 2012.

[12] AZEVEDO, R. C. D., ENSSLIN, L., JUNGLES, A. E. “A Review of Risk Management in Construction: Opportunities for Improvement”, Modern Economy, v. 05, n. 04, pp. 367-383, Apr. 2014.

[13] DUTRA, A., RIPOLL-FELIU, V. M., FILLOL, A. G., et al., "The construction of knowledge from the scientific literature about the theme seaport performance evaluation", International Journal of Productivity and Performance Management, v. 64, n. 2, pp. 243-269, Feb. 2015.

[14] LUZ, T. A., ENSSLIN, L., MUSSI, C. C., et al., "Avaliação de desempenho de serviços de tecnologia da informação: identificação do estado da arte por meio de um processo de pesquisa construtivista e análise bibliométrica”, Perspectivas em Ciência da Informação, v. 21, pp. 120-140, Apr/jun. 2016.

[15] FERNANDES, D. S., AZEVEDO, R. C., CARVALHO, E. P., et al., "A Review on the Study of Bond Behavior between Reinforcement Thin Bars and Concrete", International Journal of Science and Engineering Investigations, v. 6, n. 70, pp. 125-130, Nov. 2017.

[16] CAPES. Portal de Periódicos da CAPES, http://www.periodicos.capes.gov.br/. Acessado em setembro de 2017.

[17] CORINALDESI, V., MORICONI, G. "Influence of mineral additions on the performance of $100 \%$ recycled aggregate concrete", Construction and Building Materials, v. 23, n. 8, pp. 2869-2876, 2009.

[18] KOU, S. C., POON, C. S. "Enhancing the durability properties of concrete prepared with coarse recycled aggregate", Construction and Building Materials, v. 35, pp. 69-76, 2012.

[19] SOMNA, R., JATURAPITAKKUL, C., AMDE, A. M. "Effect of ground fly ash and ground bagasse ash on the durability of recycled aggregate concrete", Cement and Concrete Composites, v. 34, n. 7, pp. 848854, 2012.

[20] QUALIS-PERIÓDICOS. Classificação Periódicos Quadriênio 2013-2016. Plataforma Sucupira, https://sucupira.capes.gov.br/sucupira/public/consultas/coleta/veiculoPublicacaoQualis/listaConsultaGeralPer iodicos.jsf/. Acessado em agosto de 2018.

\section{ORCID}

Camila Gomes

Flávia Poggiali

Rogério Azevedo https://orcid.org/0000-0002-9812-1948

https://orcid.org/0000-0002-5882-7049

https://orcid.org/0000-0003-1276-9602 